\title{
Physiology of Sleep
}

David W. Carley ${ }^{1-4}$ and Sarah S. Farabi ${ }^{1,2}$

${ }^{1}$ Center for Narcolepsy, Sleep and Health Research, University of Illinois, Chicago, IL

${ }^{2}$ Department of Biobehavioral Health Sciences, University of Illinois, Chicago, IL

${ }^{3}$ Department of Medicine, University of Illinois, Chicago, IL

${ }^{4}$ Department of Bioengineering, University of Illinois, Chicago, IL

Corresponding author: David W. Carley, dwcarley@uic.edu

DOI: 10.2337/diaspect.29.1.5

(C)2016 by the American Diabetes Association. Readers may use this article as long as the work is properly cited, the use is educational and not for profit, and the work is not altered. See http:// creativecommons.org/licenses/by-nc-nd/3.0 for details.

IN BRIEF Far from a simple absence of wakefulness, sleep is an active, regulated, and metabolically distinct state, essential for health and well-being. In this article, the authors review the fundamental anatomy and physiology of sleep and its regulation, with an eye toward interactions between sleep and metabolism.

W hy must we sleep? Pinpointing the essential and irreplaceable aspects of sleep remains one of the great challenges of mammalian biology. Still, much has been determined about the structures, processes, and pathways underlying the regulation of sleep and the relationship of sleep to daytime functioning and overall well-being. Extensive texts on these topics are available elsewhere $(1,2)$. This article provides a concise overview of the anatomy, neurochemistry, and physiology of normal sleep and sleep homeostasis, with an eye toward the interface between sleep and metabolism. Other articles in this issue will more directly address aspects of sleep in relation to diabetes.

Generation and Maintenance of Sleep and Wakefulness

As depicted in Figure 1A, the cortical activation necessary to maintain wakefulness is supported by an extensive network of subcortical structures and pathways. Major neurochemicals of this "ascending arousal system" include excitatory norepinephrine arising from the locus ceruleus (LC), serotonin from the midline raphe nuclei, histamine from the tuberomammillary nucleus, dopamine from the ventral periacqueductal gray matter, acetylcholine from the pedunculopontine tegmentum, and the laterodorsal tegmentum of the pons and orexin from the perifornical area. Despite their apparent redundancy, normal behavioral functioning may require all of these arousing systems. For example, it is now clear that narcolepsy results from a selective loss of orexin-releasing neurons in the forebrain, accounting for the excessive daytime sleepiness, fragmented sleep, and cataplexy (sudden muscle weakness without loss of consciousness) associated with this disorder.

Initiation and maintenance of sleep require suppression of activity in the ascending arousal systems. This is accomplished by inhibitory neurons of the ventrolateral preoptic area (VLPO; Figure 1B), which remain active throughout sleep (3). The molecular "triggers" that activate the VLPO and initiate sleep onset have not been fully defined, but a substantial body of evidence points to extracellular adenosine as a candidate. Adenosine accumulates in basal forebrain during wakefulness and diminishes with ongoing sleep (4). Adenosine receptors are expressed in the VLPO and adenosine activates VLPO neurons in vivo (5), making it a reasonable candidate for the "sleep 

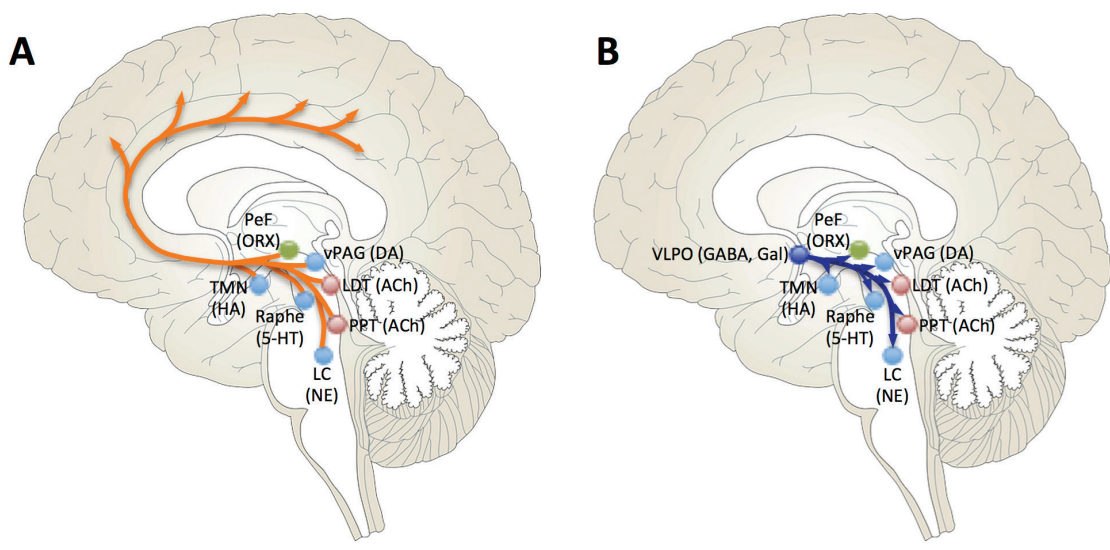

FIGURE 1. Brain networks regulating sleep and wakefulness. Panel A depicts key elements of the ascending arousal systems, with diffuse excitatory projections to the cortex. Panel B shows pathways arising from the hypothalamus that inactivate the ascending arousal system during sleep. ACh, acetylcholine; DA, dopamine; GABA, gamma amino-butyric acid; Gal, galanin; HA, histamine; LDT, laterodorsal tegmentum; NE, norepinephrine; ORX, orexin; PeF, perifornical region; PPT, pedunculopontine tegmentum; TMN, tuberomammillary nucleus; $v P A G$, ventral periaqueductal gray matter; 5-HT, 5-hydroxytryptamine.

switch." Caffeine and theophylline are potent adenosine receptor antagonists, which may form the basis for their well-known alerting effects. Despite this evidence, it is almost certain that other molecules also play important signaling roles controlling the initiation and maintenance of sleep. The monoaminergic arousal centers project to the VLPO and may serve to inhibit its activity (6). This creates the concept of "flip-flop" control of behavioral state, in which, at any given time, activity of either arousalproducing or sleep-producing neurons dominates and suppresses the other (3). In addition, the VLPO receives important circadian modulation from the suprachiasmatic nucleus - the central circadian clock (3).

Sleep itself is not a homogenous process. There exist two fundamentally distinct types of sleep: rapid eye movement (REM) sleep, which is associated with active dreaming, and non-rapid eye movement (NREM) sleep. Switches between NREM and REM sleep appear to be controlled by reciprocal inhibition between monoaminergic neurons and a specific subset of cholinergic neurons within the brainstem (7). These "REM-on" cholinergic neurons exhibit reciprocal inhibitory connections to noradrenergic (LC) and serotonergic (raphe) neurons (8). When REM sleep is triggered, REM-on cholinergic neurons become maximally active, while noradrenergic and serotonergic neurons become virtually silent. The switching between activity and inhibition of these neurons results in a characteristic cycling between NREM and REM during the sleep period.

\section{Measurement and Quantification of Sleep and Wake States}

Assessment of sleep/wake states can be made by behavioral observation, physiological monitoring, or a combination of the two. Behaviorally, sleep in adults is characterized by loss of consciousness and by relative immobility in a recumbent posture with the eyes closed. During NREM sleep, there is reduced tonus of large skeletal muscles that progresses to complete or near-complete atonia with a transition to REM sleep. Throughout sleep, there is a relative sparing of activity among respiratory pump muscles. Visual, olfactory, auditory, somatosensory, and even nociceptive sensory responses all are diminished but not eliminated during sleep (9). Furthermore, many sensory responses exhibit differing characteristics during NREM versus REM sleep.

Physiologically, the gold standard for assessment of sleep and wake states is the laboratory polysomnogram (PSG). To conduct a PSG, numerous noninvasive sensors are attached to a subject. These sensors include multiple skin electrodes, which record brain activity (electroencephalogram [EEG]), eye movements, submental muscle tone, leg movements, and electrocardiogram (ECG). Thoracic and abdominal strain gauges, oral and nasal airflow sensors, and a finger probe to measure arterial oxygen saturation are also attached to the subject to help monitor respiration during sleep.

In addition to wakefulness and REM sleep, current clinical guidelines for scoring PSGs identify three stages of progressively deepening NREM sleep: stages N1-N3 (10). These stages are recognized and scored based on characteristic rhythms and events observed in the PSG waveforms, but a detailed presentation of the scoring process is beyond the scope of this article (11). Briefly, alert wakefulness is associated with a low-amplitude mixed frequency EEG pattern. As illustrated in Figure 2, drowsy wakefulness is associated with alpha waves seen as a rhythm with peaks in the $8-$ to $13-\mathrm{Hz}$ range. Drowsiness also is associated with slow rolling eye movements that may persist into light sleep. The lightest stage of NREM sleep (N1) is characterized by a loss of alpha rhythm and presence of theta waves with a characteristic frequency of 4-7 Hz. Stage N2 sleep is marked by the expression of spindles (burst-like trains of waves in the $11-$ to $16-\mathrm{Hz}$ range with a total duration $\geq 0.5 \mathrm{sec}$ onds) and K-complexes (well-defined biphasic waves lasting $\geq 0.5$ seconds and usually maximal over the frontal cortex). Deep NREM sleep (stage 
$\mathrm{N} 3)$ is associated with large $(\geq 75 \mu \mathrm{V})$ slow $(0.5-3 \mathrm{~Hz})$ waves known as delta waves. Typically, skeletal muscle activity exhibits progressively decreasing amplitude with transitions from wakefulness to N1, N2, and N3 sleep. REM sleep is associated with the lowest skeletal muscle tone and with either sharp theta waves (sawtooth waves) or wake-like EEG patterns (Figure 2).

For scoring purposes, an overnight PSG recording is divided into 30 -second epochs, and a stage score is assigned to each epoch. Visualizing this sequence of stage scores graphically as a "hypnogram" highlights the temporal structure of the sleep process (Figure 2). During a normal night, the sleep process is cyclical, with sleep onset being followed by a rapid descent to deep stage N3 sleep within the first hour. This is followed by cyclical alternations between NREM and REM sleep occurring every 60-90 minutes throughout the rest of the night. Typically, most N3 sleep occurs during the first halfnight, whereas most REM sleep occurs during the second half-night (Figure 2). The full biological and clinical relevance of this "ultradian" cycling of sleep depth remains to be determined. PSG data also are amenable to quantitative and continuous analysis using various signal processing techniques. Because the EEG rhythms associated with differing "levels" of alertness and NREM sleep can be differentiated according to characteristic frequencies, EEG power spectrum analysis has become a very popular sleep research tool (12).

Sleep and sleep quality also can be assessed subjectively, by selfreport of the individual patient or research subject. Various mobile phone application-based, Web-based, or paper sleep and activity logs have been developed and can be useful adjuncts to laboratory or home testing for both clinical and research purposes. Although numerous standardized survey instruments have been developed to assess sleep quality,

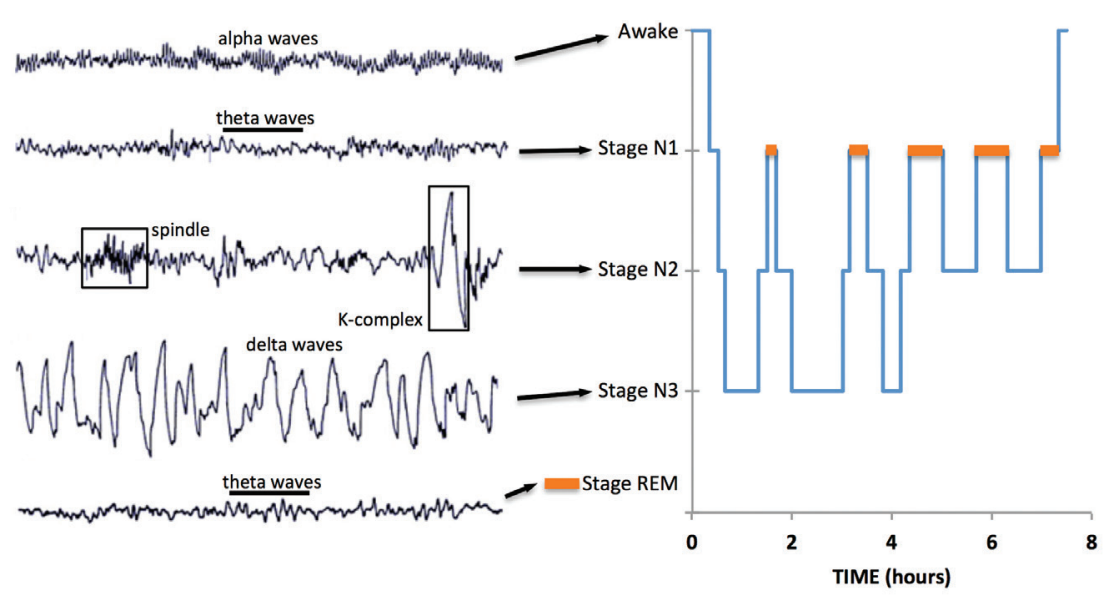

FIGURE 2. EEG features of sleep/wake stages (left) and typical temporal organization of healthy nocturnal sleep in an adult (right).

two have been very widely used: the Pittsburgh Sleep Quality Index (13) and the Functional Outcomes of Sleep Questionnaire (14).

In-home PSG testing has been performed without monitoring (15), with remote monitoring (16), and with in-home monitoring by a trained technician (17). The frequency of technically unacceptable recordings using these approaches ranges from about $10 \%$ to about $30 \%$, and it is unclear whether any consistent cost savings accrue. Numerous other methods for home sleep testing have been developed that do not incorporate EEG monitoring. The simplest of these is actigraphy, in which the subject wears a device-typically the size and shape of a wristwatch-on the nondominant wrist. The device contains multiple accelerometers and can record movements continuously for periods of up to several weeks. These movement profile data are then used to discriminate sleeping and waking periods, and their overall agreement with PSG-based determinations is good, although results of individual validation studies have varied (18). This approach has been popular in research studies, allowing collection of many days of sleep/wake patterns in a natural environment. Some actigraphic devices also contain light intensity meters and event- logging buttons, allowing subjects to note when they go to bed and when they arise. For clinical applications, "level-3" cardiorespiratory monitoring devices based on a wide array of technologies increasingly are being employed, but a thorough review is beyond the scope of this article. Typically, these devices monitor heart rate and its variability, respiratory effort and airflow, and arterial oxygen saturation. These systems are commonly used to screen for clinically significant sleep apnea syndrome (19).

\section{Endocrine Manifestations of Sleep and Wake States}

Plasma levels of most hormones exhibit significant 24-hour rhythms $(20,21)$, pointing to the importance of both the circadian clock and sleep-specific influences on their release and/ or metabolism. Some hormones are little influenced by sleep versus wakefulness, including adrenocortotropic hormone, cortisol, and melatonin; some are strongly influenced by sleep, such as thyroid-stimulating hormone (TSH) and prolactin; and some are affected by particular sleep stages, such as growth hormone (20).

Under normal conditions, prolactin levels are low during the daytime and high during sleep at night. Studies using daytime naps or sudden changes in sleep schedule have shown that sleep onset, regardless of time of 
day, is associated with a stimulation of prolactin release (22). It has been suggested that a negative association exists between EEG delta activity and pulsatile prolactin release (20).

Growth hormone also exhibits a strong sleep-dependent rhythm, with secretion being specifically associated with stage N3 sleep (20). There is a strong association between the power of EEG delta activity and the rate of growth hormone secretion during sleep (23). Furthermore, when ritanserin (a serotonin receptor antagonist) was used to augment deep sleep, the drug-related increase in EEG delta activity was associated with a proportional increase in the rate of growth hormone secretion (22). These findings strongly suggest that the mechanisms regulating delta wave production and growth hormone secretion are closely coupled, but these mechanisms have not been fully defined. This is of potential relevance to the pathogenesis and management of diabetes, because the sleep-related increase in growth hormone secretion relates directly to reduced insulin sensitivity and increased plasma glucose levels during sleep (24).

Cortisol also exhibits a significant 24-hour pattern, but, unlike growth hormone, this appears to be primarily a result of circadian influences rather than of sleep per se (20). Also in contrast to growth hormone, which peaks early during the sleep period, cortisol is at its nadir early during a nocturnal sleep period and peaks toward the end of sleep or in the early morning hours (24). There is some evidence that the sleep process does exert some inhibitory effect on cortisol release, and this may contribute to its nadir early in the sleep period. This cortisol pattern also may contribute to increased glucose levels late in the sleep period, as cortisol may inhibit insulin release (24).

TSH exhibits low daytime values, increasing during the evening and peaking around the time of sleep onset. Sleep, and especially stage N3 sleep, appears to exert an inhibitory influence on TSH secretion (20). N3 sleep is consistently associated with falling TSH levels, whereas awakenings are associated with rising $\mathrm{TSH}$ levels (25). This may be significant in diabetes pathogenesis, as TSH level has been negatively associated with various measures of insulin sensitivity. However, cause-and-effect relationships remain to be determined (26).

\section{Conclusion}

In summary, sleep is an actively regulated process significantly modulated by homeostatic influences that accumulate during ongoing wakefulness and dissipate during sleep and by circadian effects entrained to the 24hour day. Sleep has a typical underlying architecture characterized by a rhythmic alternation between NREM and REM stages, and the transitions among sleep/wake states are orchestrated by a well-defined subcortical network of brain structures. Sleep and wake states also are characterized by distinct hormonal patterns that exert potential significant influences on metabolism and glucose homeostasis.

\section{Duality of Interest}

No potential conflicts of interest relevant to this article were reported.

\section{References}

1. Billiard M. Sleep Physiology, Investigations, and Medicine. New York, Kluwer Academic/Plenum Publishers, 2003

2. Kryger MH, Roth T, Dement WC.

Principles and Practice of Sleep Medicine. St. Louis, Mo., Elsevier Saunders, 2011

3. Saper CB, Cano G, Scammell TE. Homeostatic, circadian, and emotional regulation of sleep. J Comp Neurol 2005;493:92-98

4. Strecker RE, Morairty S, Thakkar MM, et al. Adenosinergic modulation of basal forebrain and preoptic/anterior hypothalamic neuronal activity in the control of behavioral state. Behav Brain Res 2000;115:183-204

5. Chamberlin NL, Arrigoni E, Chou TC, Scammell TE, Greene RW, Saper CB.

Effects of adenosine on gabaergic synaptic inputs to identified ventrolateral preoptic neurons. Neuroscience 2003;119:913-918

6. Chou TC, Bjorkum AA, Gaus SE, Lu J, Scammell TE, Saper CB. Afferents to the ventrolateral preoptic nucleus. J Neurosci 2002;22:977-990

7. Hobson J, McCarley R, Wyzinski P. Sleep cycle oscillation: reciprocal discharge by two brainstem neuronal groups. Science 1975;189:55-58

8. Dunmyre JR, Mashour GA, Booth V. Coupled flip-flop model for REM sleep regulation in the rat. PLoS One 2014;9:e94481

9. Fontanini A, Katz DB. Behavioral states, network states, and sensory response variability. J Neurophysiol 2008;100:1160-1168

10. Iber C, Ancoli-Israel S, Chesson A, Quan S. The AASM Manual for the Scoring of Sleep and Associated Events. 1st ed. Westchester, Ill., American Academy of Sleep Medicine, 2007

11. Institute of Medicine Committee on Sleep Medicine and Research. Sleep physiology. In Sleep Disorders and Sleep Deprivation: An Unmet Public Health Problem. 2nd ed. Colten HR, Altevogt BM, Eds. Washington, D.C., National Academies Press, 2006. Available from: http://www.ncbi.nlm.nih.gov/books/ NBK19956. Accessed 18 October 2015

12. Thakor NV, Tong S. Advances in quantitative electroencephalogram analysis methods. Annu Rev Biomed Eng 2004;6:453-495

13. Buysse D, Reynolds C, Monk T, Berman S, Kupfer D. The Pittsburgh Sleep Quality Index: a new instrument for psychiatric practice and research. Psychiatry Res 1989;28:193-213

14. Weaver T, Laizner A, Evans L, et al. An instrument to measure functional status outcomes for disorders of excessive sleepiness. Sleep 1997;20:835-843

15. Kapur VK, Rapoport DM, Sanders MH, et al. Rates of sensor loss in unattended home polysomnography: the influence of age, gender, obesity, and sleep-disordered breathing. Sleep 2000;23:682-688

16. Bruyneel M, Van den Broecke S, Libert W, Ninane V. Real-time attended home-polysomnography with telematic data transmission. Int J Med Inform 2013;82:696-701

17. Carpentier N, Jonas J, Schaff J-L, Koessler L, Maillard L, Vespignani H. The feasibility of home polysomnographic recordings prescribed for sleep-related neurological disorders: a prospective observational study. Neurophysiol Clin 2014:44:251-255

18. Martin JL, Hakim AD. Wrist actigraphy. Chest 2011;139:1514-1527

19. Collop NA. Portable monitoring for the diagnosis of obstructive sleep apnea. Curr Opin Pulm Med 2008;14:525-529 
20. Gronfier C, Brandenberger G. Ultradian rhythms in pituitary and adrenal hormones: their relations to sleep. Sleep Med Rev 1998;2:17-29

21. Sadamatsu M, Kato N, Iida H, et al. The 24-hour rhythms in plasma growth hormone, prolactin and thyroid-stimulating hormone: effect of sleep deprivation. $\mathrm{J}$ Neuroendocrinol 1995;7:597-606

22. Sassin JF, Frantz AG, Kapen S,

Weitzman ED. The nocturnal rise of human prolactin is dependent on sleep. J Clin Endocrinol Metab 1973;37:436-440

23. Gronfier C, Luthringer R, Follenius $\mathrm{M}$, et al. A quantitative evaluation of the relationships between growth hormone secretion and delta wave electroencephalographic activity during normal sleep and after enrichment in delta waves. Sleep 1996;19:817-824

24. Van Cauter E, Blackman JD, Roland D, Spire JP, Refetoff S, Polonsky KS.

Modulation of glucose regulation and insu- lin secretion by circadian rhythmicity and sleep. J Clin Invest 1991;88:934-942

25. Goichot B, Brandenberger G, Saini J, Wittersheim G, Follenius M. Nocturnal plasma thyrotropin variations are related to slow-wave sleep. J Sleep Res 1992;1:186-190

26. Fernández-Real J-M, López-Bermejo A, Castro A, Casamitjana R, Ricart W. Thyroid function is intrinsically linked to insulin sensitivity and endothelium-dependent vasodilation in healthy euthyroid subjects. J Clin Endocrinol Metab 2006;91:3337-3343 